\title{
INFORMED PARENTAL CONSENT FOR COCHLEAR IMPLANTATION OF YOUNG DEAF CHILDREN: SOCIAL AND OTHER CONSIDERATIONS IN THE USE OF THE 'BIONIC EAR'.
}

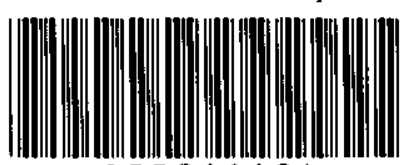

200011184

Merv Hyde and Des Power

This paper examines the social parameters surrounding the management of informed consent procedures for the parents of young deaf children for the surgical fitting of a cochlear implant ('bionic ear') to their child. Although most observers of this remarkable and well publicised medical development only see benefits in its use, the authors examine the nature of the conflict which has emergad between medical and media portrayals of the 'miracle' device and the contrasting social, cultural and linguistic views of many Deaf people. The paper analyses the components of parental consent procedures for surgical implantation of their child, covering the risk-benefit analysis and, in particular, the information base that is provided for parents about the social construction of a 'Deaf life' by many Deaf people.

\section{Deafness: a medical condition to be 'fixed' or a socio-cultural variation?}

In recent years, the fitting of cochlear implants (referred to in the popular press as the 'bionic ear') to young deaf children has become common practice. Such practice has passed beyond the 'research' stage and has reached the 'service' implementation stage, with considerable publicity and acclaim in both the professional and popular media. Most lay observers find it hard to believe that there should be any opposition to what is presented as a benefit to deaf children and their parents. Surely access to more

Merv Hyde is Associate Protessor of Special Education and Head of the School of Education and Professional Studies at Griffith University. He is also Deputy Director of the Centre for Deafness Studies and Research. Des Power is Professor of Special Education in the School of Cognition, Language and Special Education and Director of the Centre for Deafness Studies and Research at Griffith University .

Except as permitted under the Copyright Act Except as permitted under the copying this corial is prout the permission of the copyprohibited without the peive licensee or agent right owner or its exclusive licensee or agency or by way

Limited 
hearing and hence better listening, speech and personal/social and school development cannot be other than a good thing?

However, some recent commentaries (Hyde 1995; Hyde \& Power 1992), and particularly the views of Deaf communities (Australian Association of the Deaf, n.d.; Victorian Council of Deaf People 1991), have indicated that the increased fitting of cochlear implants to young deaf children, especially when accompanied by strictly 'oral-only' educational programs (instruction though listening and speech), has resulted in a conflict with the social, cultural and linguistic beliefs of signing Deaf communities. Specifically, it has been suggested that the use of cochlear implants represents an invasion of the individual rights of the deaf child by the child's parents; and that the excessive zeal demonstrated by some medical practitioners, the manufacturers of implants and the media represents a 'denial of deafness' while providing negative images of Deaf people as silent, lonely, isolated individuals, whose deafness prevents 'normal development'. Deaf people deny that this is so. Power (1992, 1997) and Hyde \& Power (1992) and others (e.g. Padden \& Humphries 1988) have reached conclusions which refute a view of deafness as an isolating and pitiable condition but describe one in which a satisfying 'Deaf life' may be constructed. Deaf community representatives see Deaf people more as a cultural and linguistic minority group (symbolised by their use of sign language) than as a disability group, only being 'handicapped' when the conditions and restrictions which the majority community institutions and attitudes put in their way make them so (Power 1992; Australian Association of the Deaf n.d.; Victorian Council of Deaf People 1991). Deaf people have been shown to be effective participants in wider society from a social and cultural context specifically associated with their role as a minority linguistic and cultural group, rather than a 'disability group' (Power 1997; Hyde \& Power 1992). This situation has been recognised in the Commonwealth's Australian Language and Literacy Policy (Dawkins 1991).

In this and some other policies, it is now recognised that signing Deaf people constitute a group like any other non-English-speaking language group in Australia, with a distinct sub-culture recognised by shared history, social life and sense of identity, united and symbolised by fluency in Auslan.

The conflict, then, would seem to be between advances in prosthetic technology and related surgical procedures that allow the use of cochlear implants within safe medical prognoses and the views of Deaf communities about their language and cultural identity. However, from the points made above, we argue that this masks a deeper conflict between descriptions of deafness (here we mean congenital deafness, not that acquired later in life) as a handicapping condition to be 'cured' or 'fixed' by 
technological advances such as the cochlear implant, versus those descriptions of deafness as a viable and enjoyable socially-constructed life (Marschark 1993).

\section{The 'battle' for parents}

The decision by an increasing number of parents of deaf infants to opt for the fitting of a 'bionic ear' has thrust them into a 'battle' with opponents of the device. Many Deaf people and their supporters deny the need to "conquer deafness' by technology (US On Line News 1999) and assert that attempts to do so are indicative of the intolerance of hearing society. They argue that to try to 'fix' deafness is like trying to 'fix' young children because of their Japanese heritage. This view is supported, to an extent, by neurologist Oliver Sacks in his influential book Seeing Voices. Sacks regards the struggle of the Deaf Community for recognition in terms of suppression by a largely ignorant hearing society. More strident critics of the use of cochlear implant technology with deaf infants have picketed medical conventions examining the use of implants with placards describing surgeons as the new 'Butchers of Belsen'.

At the heart of this sometimes bitter social debate are young deaf children and their parents. As the major decision makers, parents are, in $90 \%$ of cases, hearing and largely ignorant of the deafness-related perspectives of the issue ( $10 \%$ of deaf children have a deaf parent). Some question, therefore, how 'informed' their decision to implant their child can be, given the scientific, medical and technological impetus and media hype that surround the debate. It is often argued that the deaf children should be old enough to be able to decide for themselves. This argument is countered by the proponents of the implant who refer to the need to intervene with very young deaf children (as young as 18 months) to capture the child's neurologically critical period for the development of spoken language.

Many members of Deaf communities and some educators of the deaf have therefore questioned the situation whereby parents are able to give consent for their young deaf child to receive a cochlear implant. This questioning has, inter alia, focused on the issue of how 'informed' parental consent can be under the present procedures used by cochlear implant fitting services, which tend to use the 'medical model' of deafness as a condition to be cured, rather than the 'social/cultural model' of a viable life as a Deaf person.

\section{Procedures used to obtain 'informed consent'}

Examination of the process of informing parents for gaining consent to cochlear implants for young deaf children is therefore the focus of this paper. We are not here concerned with whether young deaf children should be implanted or not; that is a separate issue. We examine here only the issue of whether parents are given full 
enough information about all aspects of deafness: sufficient to give fully informed consent to the procedure. Other work in progress of the present writers examines the outcomes of early implantation in terms of school and 'real life' outcomes and Power and Leigh (in preparation) examine claims that access to signing will prevent or lessen the oral skills development of deaf children and demonstrate that there is little empirical evidence to support such claims. It is the view of the present writers that cochlear implant teams need to be more accepting of the value of a 'Deaf life' and the utility of signing in the development of deaf students and that the signing Deaf community needs to become more accepting of those children who have been implanted; it was usually not their decision to have the procedure.

\section{What is informed consent?}

In the therapeutic setting, informed consent has its beginnings in the moral principle of respect for the autonomy of the person making the decision. The focus is on the individual's right to authorise or deny a particular surgical intervention. The autonomous person should not be hindered in his or her understanding, reasoning or decision-making and should be able to make a decision in a context free from constraint or undue influence. They must have an appropriate level of understanding of the information presented. In the Australian and similar legal systems the law has translated this moral principle of informed consent into a legal duty to warn of the risks of having or not having the treatment. More is involved than risk management and the minimisation of possible claims for negligence. The process of communication underpins informed consent in the therapeutic setting, and needs to be individualised to ensure that the decision-maker is able to give valid and meaningful consent.

Legally and ethically, it is clear that parents can provide consent for conditions involving their children. This is particularly the case for conditions of a life-threatening nature. Increasingly, however, parents are asked to provide informed consent for conditions which are not of a life-threatening nature. Perhaps the forerunner of this form of consent in many Western societies has been the issue of circumcision of young boys, and, in other societies, of young girls. Neither of these conditions is considered life-threatening, yet informed parental consent has become standard practice before such procedures are undertaken.

Some commentators suggest that in the case of cochlear implants we are in a position of not really being able to say 'no' to parents who believe they have informed themselves on the topic and the perceived benefits the implant may have for their young deaf child. One representative of an Australian implant program, for example, has written to us that 'An ethical problem occurs when all the [professionally] informed advice has been given and a parent insists on going ahead. If the child meets the audiological criterion (the measured degree of deafness) it may not be legally possible 
to deny the parents their right to make the decision on behalf of the child.' The issue of parents' right to make decisions about the physical, intellectual and personal characteristics of their planned infant will be further broadened by the coming debate on the selection of children's attributes via genetic engineering (for the case of deafness, see Power 1997). It has already been suggested (e.g. in the film Gattaca) that refusal to accept a genetically engineered fate may become illegal. More realistically, Tucker (1998) - herself a congenitally deaf recipient of an implant as an adult - has suggested that if deaf people (or their surrogates, parents) refuse potentially ameliorating treatments of disabilities (i.e. refuse to have an implant fitted), it is possible that public funding support for the 'treatment' of their deafness by special education might not be made available in the future. After reviewing a number of cases where American courts have denied compensation to people with potentially alleviable disabilities who have refused to have procedures that would have alleviated that disability, and citing the much lower costs of regular versus special education placement (i.e. the higher expense of special education which she claims would not be necessary for implanted deaf children), she says:

It seems likely that in the future more courts will hold that the law does not require that an individual with a physical impairment be provided with accommodations which would not be necessary if the individual would obtain reasonable medical treatment that would obviate the need for such accommodations ... Members of the public, including politicians, are likely to ask: why should the public and private sectors be required to spend money to provide accommodations for a person whose disability is correctable, when correcting the disability would in itself help to level the playing field for that person? (Tucker 1998: 12)

It may clearly become crucial what 'reasonable medical treatment' is (an implant?); and whether a disability is 'correctable' (via an implant?). How are these terms going to be defined and who is going to define them? We are playing for very high stakes indeed in terms of ethical decisions and policy determination and implementation.

Parents' authority to give consent to medical treatment exists only to the extent that their child is incapable of giving valid consent. It has been established in Australian law that a child under the age of eighteen years can consent to medical treatment when that child has sufficient intelligence and maturity to understand the nature and consequences of the particular treatment. At this stage of our analysis, we are not aware of any procedures where the opinion of the child is included in the cochlear implant decision-making process, although some examples may exist for older deaf children. From the same implant program as quoted above: 'In this latter regard [i.e. the giving of consent] we would regard the child's view as an important factor. However 
since most of the candidates are very young this issue does not normally arise. It might arise, however, if the parents of a 12 year old wished to pursue the option [of an implant]. In this case the issue would need to be discussed with the young person as well.' It would seem that in most cases that the fitting of an implant to a child at an early age to realise neurologically optimal conditions for auditory development means that an informed contribution from the child is not possible.

Legal definitions of informed consent have focused on areas like the sterilization of young intellectually disabled individuals (especially young women) or on the ethics of cessation of life-sustaining treatments for significantly disabled neonates. Various legal and ethical safeguards have been developed in these areas to guard against the possibility of unethical practice, even by the legal guardians of such children. In these cases, the procedure under consideration can be said to be physically invasive (as in the case of sterilisation) or life-threatening (as in the case of withdrawal of life support systems). It has been argued, however, that the decision to fit a cochlear implant does not parallel these examples. The cochlear implant is claimed to be a prosthesis that produces a significant benefit for the recipient, with negligible risk. It is this balance between 'benefit' and 'risk' which is at the heart of the informed consent debate about fitting cochlear implants.

\section{A risk/benefit analysis}

The documentation on cochlear implant programs available to us suggests that the 'risk' is considered chiefly in a medical/surgical context that can be clearly defined and explained to parents of deaf children. Examination of the documents explaining the conditions for candidature from two of Australia's implant programs reveals that the informed consent documents provided to parents focus almost exclusively on issues of risk on the medical model. It is evident from analysis of these and similar documents (for example, those used by some cosmetic surgeons) that the issue of risk is usually defined by criteria that may be covered by insurers to protect the medical practitioners involved in case of accident, negligence or unsatisfactory outcomes during or following the procedure. ' None of the apprehended 'risks' mentions the possibility of unsatisfactory outcomes in the personal, linguistic or auditory sphere beyond a typical: 'PLEASE NOTE that we cannot accurately predict, nor guarantee, the level of speech recognition that an individual will gain from the cochlear implant procedure.' (Emphases in original.) This is not to suggest that the documents which seek to inform parents are limited by what is insurable, but clearly that is the central issue when determining risk for the medical practitioners providing cochlear implantation procedures. This then colours the notion of 'informed consent' and in many cases, because of the medical model orientation of cochlear implant teams, limits the information given to parents about the alternative social and cultural models of deafness. The 'risks' outlined in the 'Possible Risks' statement are, on the face of it, 
quite daunting, and it seems likely that the tone of the advice to parents contemplating an implant for their children is much more optimistic than these statements would imply on paper.

In view of the fact that a 'Deaf Life' (Power 1997) can be a viable and satisfactory one without cochlear implant technology it is reasonable to suggest that the risk/ benefit model of informed consent for parents of deaf children should be broadened to include the social, linguistic and cultural characteristics associated with being Deaf: namely that, in contrast to the perception that there is disadvantage in being deaf, the 'viable Deaf life' alternative should be presented to parents. Some have even argued that there is a disadvantage to young deaf children in their being directed into a (likely imperfectly accessible) 'hearing' world through implantation and thus not being able to fully access the rich personal, social, cultural and linguistic context that membership of the Deaf community could provide. It is argued that parents of deaf children should have such information available if they are truly to be informed about the futures available for their child.

In none of the informed consent procedures we analysed was this issue canvassed. The informed consent procedures were determined by the medical provider, administered by the medical provider and final authorisation for fitting managed by the medical provider, with consideration only of the medical parameters of benefit and risk, not the personal, social or educational ones. It does not appear that any deaf adults as models of what is possible as a "deaf' person are presented to parents at this crucial decision stage.

Further, it is also argued that the developmental benefits frequently claimed to be associated with fitting implants to young deaf children have not been documented in the literature of the field in ways that would support some of the claims made by their protagonists. Most reported measures are of narrowly conceived perceptual outcomes. Very few studies of social, linguistic or educational development have been reported to demonstrate the general academic and 'life-style' benefits frequently claimed by implant supporters. While there are some outstanding cases anecdotally presented in the professional literature and by the media, there are also many cases where there is not a clear benefit for the recipient over what might have been expected from the use of a conventional hearing aid, much less the digital programmable aids now coming on stream. As Parr (1991) has also pointed out, very few wearers of conventional aids ever obtain the level of intensive training provided to children with implants, and it is arguable that were conventionally aided children to get that level of support, they would do as well as most of the children now being implanted. Whether the very variable response benefit pattern associated with implantation is presented to parents is doubtful, beyond the vague disclaimer quoted above. 
Currently the description of 'benefit' and 'risk' remains up to the providers of the service. There is therefore a potential conflict between the aspirations of the service providers to promote the benefits of implants and their requirement to provide detailed and understandable information on which a decision to proceed or not will be based.

\section{Summary and conclusions}

In summary, therefore, the present debate would seem to rest at a point where there are high medical, public/media, and hence parental, expectations of the 'normalising' possibilities of cochlear implantation of very young deaf children, associated with high perceptions of benefit to the child's speech, learning and personal/social development. It would also seem, however, that the notion of informed consent is not a broadly defined one. The current practice of informing the parents of the child patient operates within a medical model and is indicative of the way in which clinical practice may at times disregard wider social and developmental issues. Provision of information to parents to enable informed consent tends to be based on narrowly conceived medical criteria, not on a broader consideration of social and cultural factors pertaining to the development of a Deaf life. The information provided does not contain any descriptions of disadvantage that may occur for a young deaf child as a result of a parental decision to have a cochlear implant fitted. The medical model, particularly as reported in the media, tends to characterise Deaf Culture as a sad or unfortunate compensation for a profound hearing disability. Those currently excluded from candidature for implant programs are often described as being untreatable and, as such, 'restricted' to the use of sign language. Since both the doctor and the parents are usually hearing, and they agree that hearing is a blessing, the child is not consulted nor are deaf adults presented to provide an alternative view of deafness.

Of course, given our research knowledge about early life sensory deprivation and human development, it would be inappropriate to ask the most likely candidates young deaf children - to provide their consent. There is, however, a significant number of children over ten who have been fitted with implants in Australia. Given similar precedents in Australian law, one could question whether the views and understanding of those children were inccrporated into the informed consent procedures. In addition, one could question whether such implantation of children well beyond a neurologically optimal period is appropriate, or even cost effective. This is another, less frequently discussed but equally important, dimension of the risk/benefit paradigm.

However, as stated earlier, it is not possible to deny parents their right to make such a decision. The issue continues to focus on the information base upon which they make it. A particular question that needs to be answered is, "How do we know that the parent or the guardian is fully informed?' This is a very difficult question to answer and one suggestion has been that the informed consent procedure should be conducted 
in a non-directional independent counselling context; that is, one that involves removing the service provider from the counselling/advice context because of potential conflict of interest or narrowness of view, and providing all relevant facts to facilitate parental understanding and decision making through an independent authority. This would seem to be a reasonable way forward.

Present practice, as we have seen, seems to be bound within a medical context and to focus more on those criteria that are medically liable to negligence claims. The outcomes of 'benefit' (phonologically, auditorially, linguistically and socially) are clearly variable in the cases to date, and the definition of 'risk' (beyond the risks described in a medical context) needs to be expanded, particularly in social and cultural terms. The level of the material presented and the way in which it is presented to parents are also factors to be considered when determining how informed the ultimate decision can be. The inclusion of descriptions of Deaf life and of the features of the Deaf community should be added to the risk/benefit descriptions and Deaf people with intimate knowledge of life as a deaf person included in the advisory teams. Finally, the central role of the medical provider in documenting, providing and monitoring the informed consent procedure may lead to a perceived conflict of interest for what is not a life-threatening condition and where the ultimate judgment is one of benefit to the individual.

As Moores (1995: 256, emphasis in original) has said:

Surgical procedures should be demonstrably superior to any other altemative before we resort to them. The demonstration of superiority should not be left entirely to those with a vested interest in the success of the procedure. Parents have a legal and moral right to make decisions regarding the welfare of their children. We have the moral obligation to investigate all issues completely and present complete and honest information to parents in a professional manner to the best of our ability.

Consideration of the general principles of understanding of 'disability' highlighted by the case of informed consent for cochlear implantation may well foreshadow many such debates in the not-far-distant future. These debates may be centred around genetic engineering and other such procedures for non-life-threatening states rather than around surgery, but an informed public needs to be aware of the issues surrounding who decides what a 'viable life' is; and what interventions may be legitimate in 'normalizing' lives perceived by some (e.g. Deaf people) to be 'normal' within a different understanding of normality, despite a general public and some professional opinion to the contrary. These are issues of broad social policy that need informed public opinion. Who is to be valued (even if 'different') is of great consequence to 
individual lives and the community generally. Social values, not technology, should drive this decision process.

\section{Endnote}

1. Typical 'Possible Risks' statement from an Australian Cochlear Implant Program:

1. Inability to place some or all of the electrodes inside the cochlea.

2. Infection at the site of the surgery, perhaps requiring removal of the device.

3. Leakage of fluid from the cochlea, perhaps requiring a further operation.

4. Electronic failure of the cochlear implant.

5. Injury to the facial nerve ... causing either temporary or permanent facial 'weakness'.

6. Disturbance of taste.

7. Giddiness.

8. Increased tinnitus

9. Unsuccessful electrical stimulation, due to insufficient hearing nerve fibres.

10. Some electrodes may stimulate non-hearing nerves causing pain or discomfort.

11. Should there be a need for the patient to undergo Magnetic Resonance Imaging ... the magnet in the cochlear implant would need to be surgically removed.

- In addition to the above, some risks are involved in general anaesthesia: chest, heart and brain complications may occur.

- The procedure may also involve risks that are currently not known.

- No compensalion will be provided as no more than minimal risk is involved.

\section{References}

Australian Association of the Deaf (n.d.) Policy on the Cochlear Implant, Petersham, Australian Association of the Deaf.

Dawkins, J. (1991) Australia's Language: the Australian language and literacy policy, Canberra, AGPS. 
Hyde, M.B. (1995) 'Ethical dimensions of cochlear implantation of deaf children', Annals of Otology, Rhinology and Laryngology: Supplement on Cochlear Implants, 19-20.

Hyde, M. B. \& Power, D. J. (1992) 'The use of Australian Sign Language by deaf people', Sign Language Studies 75, 167-82.

Marschark, M. (1993) The Psychological Development of Deaf Children, New York, Oxford University Press.

Moores, D. F. (1995) 'Cochlear implants and hearing aids', American Annals of the Deaf, 140, 245-46.

Padden, C. \& Humphries, T. (1988) Deaf in America, Cambridge, Mass., Harvard University Press.

Parr. W. (1991) 'Deafness in children' (letter to the editor), Medical Journal of Australia, 154, 490-91.

Power, D. (1992) 'Deaf people: a linguistic and cultural minority community or a disability group?' Australian Disability Review 4-92, 43-48.

Power, D. (1997) Constructing Lives: The Deaf Experience, Brisbane, Griffith University Faculty of Education.

Power, D. J. \& Hyde, M. B. (1992) 'The Deaf Community and cochlear implants', Medical Journal of Australia, 157, 421-22.

Sacks, O. (1998) Seeing Voices: a journey into the world of the deaf, New York, HarperCollins.

Tucker, B. T. (1998) 'Deaf culture, cochlear implants, and elective disability', Hastings Center Report, 28 (4), 6-14.

US On Line News (March, 1999), http: // www. usnews.com/ usnews/issue/990301/ ldeaf .htm.

Victorian Council of Deaf People (1991) Cochlear Implant Policy, Melbourne, Victorian Council of Deaf People. 